\title{
MODELAGEM, SIMULAÇÃO E VALIDAÇÃO DE METAMODELO DE UMA PLANTA DE TRATAMENTO DE EFLUENTES ATRAVÉS DA TÉCNICA KRIGING
}

\author{
V. M. C. ALVES 1 , F. S. LIMA ${ }^{1}$, P. L. BARROS ${ }^{1}$ e A. C. B. de ARAUJO ${ }^{1}$ \\ ${ }^{1}$ Universidade Federal de Campina Grande, Unidade Acadêmica de Engenharia Química \\ E-mail para contato: victor.alves@eq.ufcg.edu.br
}

\begin{abstract}
RESUMO - Os avanços na modelagem computacional de processos químicos permitiram a engenharia a obtenção de resultados cada vez mais confiáveis, baseados em modelos rigorosos, os quais partem da fundamentação teórica oriunda de princípios como o de conservação da massa e da energia. Todavia, tais modelos requerem geralmente um maior esforço computacional, devido à sua complexidade. Com isso, existe a necessidade da geração de modelos reduzidos, baseados em aproximações do modelo rigoroso, denominados metamodelos. A vantagem da construção de metamodelos para substituição parcial ou total dos modelos rigorosos originais está no ganho de tempo na obtenção de resultados e na redução do esforço computacional, aspectos importantes na modelagem de processos químicos. O presente trabalho propõe-se a modelar, simular e validar um metamodelo de um processo químico de alta complexidade e altamente não-linear: $O$ setor reacional de uma unidade de tratamento de efluentes. O metamodelo foi gerado através da técnica da Kriging. Os resultados obtidos no metamodelo foram comparados com o modelo original no que diz respeito à qualidade do efluente e ao custo anual da planta. Os resultados apresentaram-se altamente satisfatórios, ao executar uma validação através do método de análise de variância entre as variáveis de saída do modelo original e o metamodelo, com $99 \%$ de confiança.
\end{abstract}

\section{INTRODUÇÃO}

Com o aumento da preocupação da questão ambiental nos últimos anos, gerando regulamentos cada vez mais rigorosos para a questão do tratamento de efluentes, é evidente que a manipulação correta e eficaz desses sistemas que possuem uma alta não-linearidade e que sofrem altas perturbações no influente de entrada, como observado por Jeppsson et al. (2011), se faz necessária. Além disso, A medida que a tecnologia avança, melhorando a eficácia das plantas reais, muitas vezes os modelos tradicionais tornam-se demasiadamente complexos, o que atrasa a convergência de resultados e diminui a eficácia do estudo, pesquisa e investigação de tais sistemas.

Portanto, é necessária a construção de modelos que sejam capazes de representar os processos químicos com o máximo de precisão possível sem perder a velocidade de obtenção de resultados, que é onde se encaixam os metamodelos. Assim, pode-se afirmar que construção de metamodelos estão associadas, geralmente, a situações onde o esforço 
computacional, o tempo para obtenção dos resultados e a confiabilidade dos resultados são aspectos conflitantes, porém vitais. (Gomes, 2007).

\section{FUNDAMENTAÇÃO TEÓRICA}

A modelagem da unidade de tratamento de efluentes, utilizada neste trabalho como modelo rigoroso-base para construção do metamodelo kriging, é a proposta por Alex et al. (2008) e Jeppsson et al. (2011). O modelo consiste em um reator de lodo ativado composto por cinco compartimentos, onde os dois primeiros são anóxicos e os três últimos aerados. É considerada uma vazão média de efluente de $18446 \mathrm{~m} / \mathrm{d}$. O sistema possui um volume total de $12000 \mathrm{~m}^{3}$, onde $6000 \mathrm{~m}^{3}$ corresponde ao volume total do reator de lodo ativado e $6000 \mathrm{~m}^{3}$ para o sedimentador. A vazão de descarte corresponde a $385 \mathrm{~m}^{3} / \mathrm{d}$.

Figura 1 - Layout geral da BSM1. Adaptado de Jeppsson et al. (2011)

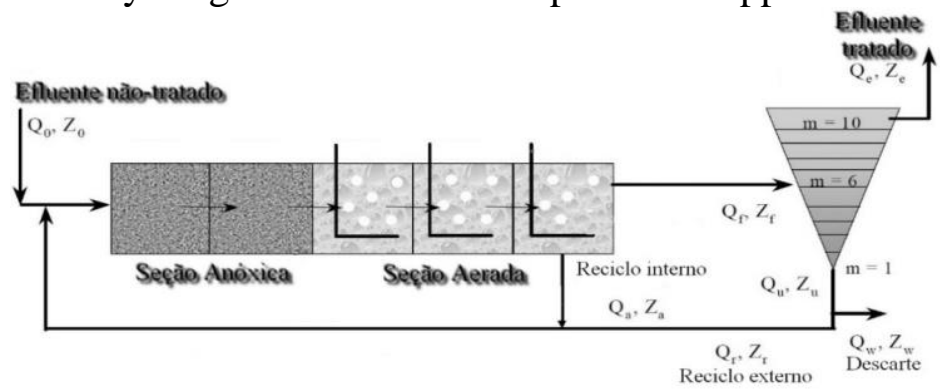

\subsection{METAMODELOS}

Definem-se como metamodelos, segundo Palmer e Realff (2002) como funções matemáticas simplificadas, baseadas em dados que foram obtidos através de uma simulação computacional com um modelo rigoroso. O objetivo de um metamodelo é substituir o modelo original em casos onde este demanda um esforço computacional elevado ou segundo Gomes (2007), quando o modelo rigoroso apresenta limitações que dizem respeito a sua confiabilidade, tal como problemas de convergência em rotinas iterativas.

\subsection{PLANEJAMENTOEXPERIMENTAL - O HIPERCUBO LATINO}

Neste trabalho, a técnica de planejamento experimental utilizada entra na categoria das técnicas de preenchimento de espaço (space filling). Essa categoria de técnicas parte da hipótese que as características importantes do modelo original (rigoroso) estão distribuídas uniformemente no espaço experimental com a mesma probabilidade, como observado por Gomes (2007). Entre essas técnicas, uma das mais famosas e usadas é a amostragem por hipercubo latino (latin hypercube sampling).

Figura 2 - Hipercubo latino em projeto bidimensional. Fonte: Forrester (2008)

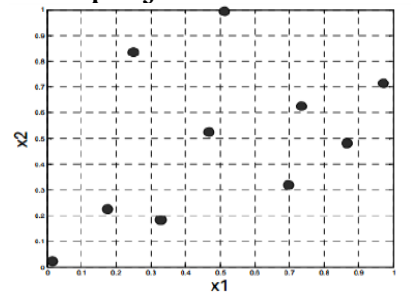


Observa-se que os pontos são distribuídos pelos subintervalos do espaço de projeto de tal forma que eles não se repetem nas linhas e colunas, aparecendo apenas uma vez em cada.

\subsection{METAMODELOS KRIGING}

Os metamodelos kriging pertencem a uma categoria maior de funções matemáticas denominadas modelos de superfície. A premissa teórica principal da metamodelagem por krigagem baseia-se que pontos próximos entre si em um espaço qualquer, possuem valores mais semelhantes do que pontos que estejam mais afastados entre si. Esse princípio baseia-se no princípio da geoestatística.

A fundamentação matemática a seguir do método Kriging é a mais usada utilizada na literatura, conforme os estudos de Lophaven et al. (2002). Inicialmente, considera-se um conjunto de funções $\mathrm{Yi}(\mathrm{X}, \mathrm{P})$, que representa um modelo matemático:

$Y_{I}=Y_{I}(X, P)$

Onde $\mathrm{X}$ são as variáveis independentes do sistema, também chamados de pontos de projeto e $\mathrm{P}$ os parâmetros. Assim, a predição via Kriging que se aproxima do modelo rigoroso é dado por:

$\widehat{Y}_{i}=\tau_{i}(x)+z_{i}(x)$

Onde as funções $\tau$ são modelos de regressão dados por:

$\tau(\beta, x)=\sum_{k=1}^{k=n F} \beta_{k} f_{k}(x)$

As funções $f$ são funções atribuídas de forma que o modelo de regressão $\tau$ seja polinomial. Já para o segundo termo do metamodelo kriging, $\mathrm{z}(\mathrm{x})$, este é denominado de termo de correlação, e são baseadas em funções randômicas gaussianas, de acordo com Santner (2003). A covariância entre dois pontos quaisquer do ponto de projeto $\mathrm{X}$, se correlaciona com a função de correlação $\mathrm{Z}$ da seguinte forma:

$E[z(w), z(x)]=\sigma^{2} \mathcal{R}(\theta, w, x)=\operatorname{cov}(w, x)$

Onde $\mathrm{R}$ é a matriz de correlação entre os dois pontos em questão e $\theta$ um vetor de parâmetros. De maneira geral para um vetor qualquer, a matriz de correlação, para o modelo da família exponencial por exemplo, é dada por:

$$
\begin{gathered}
\operatorname{cor}\left[Y\left(x^{i}\right), Y\left(x^{1}\right)\right]=\exp \left(-\sum_{j=1}^{k} \theta_{j}\left|x_{j}^{i}-x_{j}^{1}\right|^{p}\right) \\
\mathcal{R}=\left(\begin{array}{ccc}
\operatorname{cor}\left[Y\left(x^{1}\right), Y\left(x^{1}\right)\right] & \cdots & \operatorname{cor}\left[Y\left(x^{1}\right), Y\left(x^{n}\right)\right] \\
\vdots & \ddots & \vdots \\
\operatorname{cor}\left[Y\left(x^{n}\right), Y\left(x^{1}\right)\right] & \cdots & \operatorname{cor}\left[Y\left(x^{n}\right), Y\left(x^{n}\right)\right]
\end{array}\right)
\end{gathered}
$$

\section{METODOLOGIA}

Sacks et al. (1989) descreveu a metodologia para geração de metamodelos e nomeou a mesma pelo acrônimo DACE (Design and Analysis of Computer Experiments). A metodologia básica para geração de um metamodelo consiste em: 
- Seleção de um planejamento experimental para gerar os dados de entrada;

- Alimentação dos dados de entrada no modelo rigoroso, com o objetivo de gerar as saídas do modelo rigoroso (experimento computacional)

- Alimentação dos dados de entrada gerados pelo planejamento experimental e dos dados de saída gerados pelo modelo rigoroso, no algoritmo que irá gerar o metamodelo;

- Validação do metamodelo, fazendo-o avaliar variáveis-resposta diferentes das que foram utilizadas em sua construção, e comparar essas respostas com o modelo rigoroso (validação cruzada). Esta técnica é amplamente utilizada na literatura, como pode ser visto em Santner (2003), e o comportamento esperado é o linear entre as variáveis.

A escolha das variáveis de entrada seguiu a modelagem original de Alex et al. (2008) e Jeppsson et al. (2011), conforme a tabela 1:

Tabela 1 - Variáveis de Entrada do modelo rigoroso consideradas no metamodelo kriging.

\begin{tabular}{|c|c|c|c|c|}
\hline Variável & Descrição & Unidades & Classificação & $\begin{array}{l}\text { Limites (unidades } \\
\text { da variável) }\end{array}$ \\
\hline $\mathbf{S}_{\text {I }}$ & Matéria Orgânica inerte solúvel & gDQO. $\mathrm{m}^{-3}$ & Distúrbio & $+-10 \%$ do nominal \\
\hline Ss & $\begin{array}{l}\text { Matéria Orgânica rapidamente } \\
\text { biodegradável (solúvel) }\end{array}$ & gDQO. $\mathrm{m}^{-3}$ & Distúrbio & $+-10 \%$ do nominal \\
\hline $\mathbf{X}_{\mathbf{I}}$ & Matéria Orgânica particulada & gDQO. $\mathrm{m}^{-3}$ & Distúrbio & $+-10 \%$ do nominal \\
\hline $\mathbf{X}_{\mathbf{S}}$ & $\begin{array}{l}\text { Matéria Orgânica lentamente } \\
\text { biodegradável (particulada) }\end{array}$ & gDQO. $\mathrm{m}^{-3}$ & Distúrbio & $+-10 \%$ do nominal \\
\hline $\mathbf{X}_{\mathbf{B}, \mathbf{H}}$ & Biomassa Heterótrofa & gDQO. $\mathrm{m}^{-3}$ & Distúrbio & $+-10 \%$ do nominal \\
\hline $\mathbf{X}_{\mathbf{B}, \mathbf{A}}$ & Biomassa Autótrofa & gDQO. $\mathrm{m}^{-3}$ & Distúrbio & $+-10 \%$ do nominal \\
\hline $\mathbf{X}_{\mathbf{P}}$ & $\begin{array}{l}\text { Matéria Orgânica particulada } \\
\text { resultante da morte microbiana }\end{array}$ & gDQO. $\mathrm{m}^{-3}$ & Distúrbio & $+-10 \%$ do nominal \\
\hline So & Oxigênio dis solvido & gDQO. $\mathrm{m}^{-3}$ & Distúrbio & $+-10 \%$ do nominal \\
\hline $\mathbf{S}_{\mathrm{NO}}$ & Nitrato e Nitrito & $\mathrm{gN} \cdot \mathrm{m}^{-3}$ & Distúrbio & $+-10 \%$ do nominal \\
\hline $\mathbf{S}_{\mathrm{NH}}$ & Amônia e amônio & gN.m ${ }^{-3}$ & Distúrbio & $+-10 \%$ do nominal \\
\hline $\mathbf{S}_{\text {ND }}$ & Nitrogênio orgânico solúvel & gN.m ${ }^{-3}$ & Distúrbio & $+-10 \%$ do nominal \\
\hline $\mathbf{X}_{\text {ND }}$ & $\begin{array}{l}\text { Oxigênio orgânico particulado } \\
\text { (insolúvel) }\end{array}$ & $\mathrm{gN} \cdot \mathrm{m}^{-3}$ & Distúrbio & $+-10 \%$ do nominal \\
\hline TSS & Sólidos suspensos totais & gSS.m $\mathrm{m}^{-3}$ & Distúrbio & $+-10 \%$ do nominal \\
\hline $\mathbf{Q}$ & Fluxo volumétrico & $\mathrm{m}^{3} \mathrm{~d}^{-1}$ & Distúrbio & $+-10 \%$ do nominal \\
\hline $\mathbf{T}$ & Temperatura & ${ }^{\mathrm{O}} \mathrm{C}$ & Distúrbio & $+-10 \%$ do nominal \\
\hline $\mathbf{Q}_{\mathbf{a}}$ & Reciclo interno & $m^{3} d^{-1}$ & MV & $30972-103240$ \\
\hline $\mathbf{Q}_{\mathbf{r}}$ & Reciclo externo & $\mathrm{m}^{3} \mathrm{~d}^{-1}$ & MV & $10324-41296$ \\
\hline $\mathbf{Q}_{\mathbf{w}}$ & Descarte & $\mathrm{m}^{3} \mathrm{~d}^{-1}$ & MV & $320-420$ \\
\hline K⿺а3 & $\begin{array}{c}\text { Coeficiente de transferência de massa } \\
- \text { compartimento } 3\end{array}$ & $d^{-1}$ & MV & $80-100$ \\
\hline $\mathbf{K}_{\mathbf{l}} \mathbf{a} 4$ & $\begin{array}{c}\text { Coeficiente de transferência de massa } \\
\text { - compartimento } 4\end{array}$ & $d^{-1}$ & MV & $80-100$ \\
\hline $\mathbf{K} \mathbf{a} 4$ & $\begin{array}{c}\text { Coeficiente de transferência de massa } \\
- \text { compartimento } 5\end{array}$ & $d^{-1}$ & MV & $80-100$ \\
\hline
\end{tabular}

Os limites para as variáveis manipuladas são os de Jeppsson et al. (2011) e foram respeitados para manter a fidelidade ao modelo original. Para os distúrbios de entrada na planta (vazões, concentrações de contaminantes, biomassa, substratos e temperatura) foi adotado um desvio de $+-10 \%$ dos valores nominais presentes também em Jeppsson et al. (2011). 


\section{RESULTADOS}

Foram construídos metamodelos kriging com diversas funções de regressão e correlação, e o que obteve melhor ajuste foi o que combinou um modelo de regressão quadrática com um modelo de regressão gaussiana, conforme pode ser visto na figura 1, resultado gráfico das variáveis de saída do modelo original de Alex et al. (2008) e Jeppsson et al. (2011) versus as variáveis de saída previstas pelo metamodelo kriging. As variáveis de saída correspondem à demanda química e biológica de oxigênio (DQO e DBO em gDQOm ${ }^{-3} \mathrm{e}$

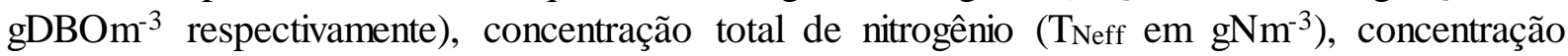
de amônia total $\left(\mathrm{SNH}_{\mathrm{eff}} \mathrm{em} \mathrm{gNm}^{-3}\right)$, teor de sólidos suspensos $\left(\mathrm{TSS}_{\text {eff }} \mathrm{em} \mathrm{gSSm}^{-3}\right.$ ) e custo total da planta (OCI em $\mathrm{kWh} / \mathrm{d}$ ), todos relativos ao efluente após o tratamento. Essas são as principais variáveis e mais importantes de processos de tratamento de efluentes, pois tratamse de medições efetuadas na realidade por órgãos de inspeção ambiental e o custo, trata-se do gasto da planta em questão. Sendo assim estas as melhores variáveis a ter o desempenho analisado no metamodelo kriging. As modelagens da estimação de tais variáveis podem ser apreciadas nos trabalhos de Alex et al. (2008) e Jeppsson et al. (2011).

Figura 3 - Comparação entre as saídas do modelo original versus metamodelo kriging.
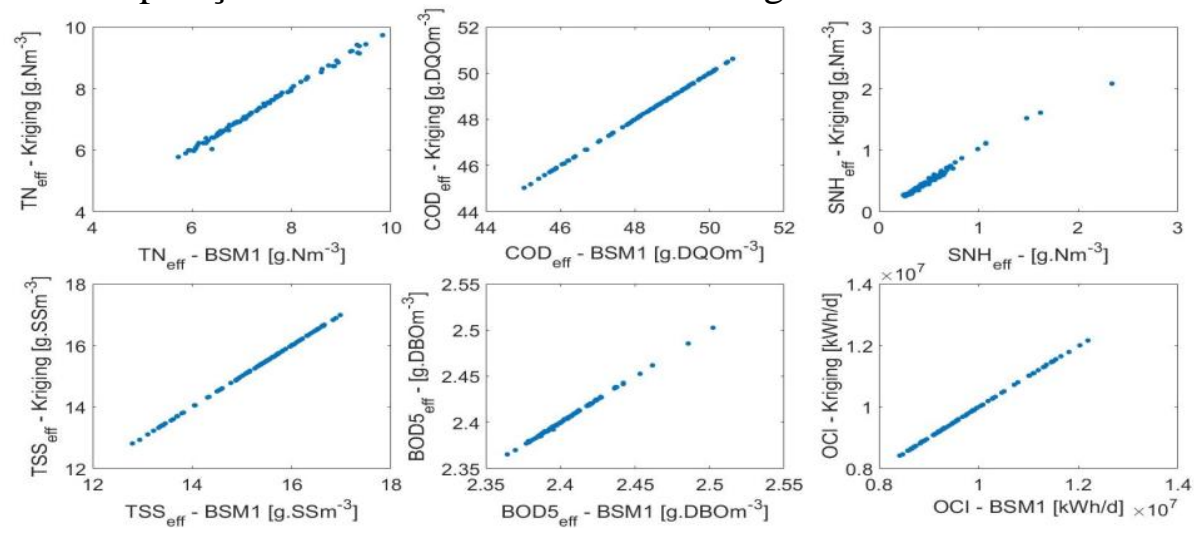

Outra comparação efetuada além do método da validação cruzada, foi a utilização de uma análise de variância multivariada (MANOVA), onde foram utilizados os três principais testes: Teste de Wilk, Layley-Hottelling e Pillai, para um nível de confiança de 99\%. Foram analisadas as saídas do modelo rigoroso versus as saídas do metamodelo kriging. Os resultados constam na tabela 2 :

Tabela 2 - Resultados de testes estatísticos de análise de variância multivariada (MANOVA)

\begin{tabular}{|c|c|c|}
\hline Critério & Estatística & P \\
\cline { 3 - 3 } do Teste & \\
\hline Lawley- \\
Hotelling & 0,00023 & 0,998 \\
\hline Pilllai's & 0,00023 & 0,998 \\
\hline Wilk's & 0,99977 & 0,99 \\
\hline
\end{tabular}

É possível observar que os testes confirmam a teoria de igualdade estatística entre o metamodelo kriging construído neste trabalho e o proposto originalmente nos trabalhos de Alex et al. (2008) e Jeppsson et al. (2011), uma vez que a potência dos três testes (P) foi superior ao erro admitido ( $1 \%$ ou 0,01$)$. 


\section{CONCLUSÕES}

O metamodelo gerado pela técnica kriging foi capaz de mimetizar com sucesso o comportamento da modelagem original proposta por Alex et al (2008) e Jeppsson et al. (2008), sendo um candidato em potencial à substituição do modelo original em casos onde a obtenção dos resultados necessite ser efetuada de forma rápida, eficaz e robusta, além para fins de otimização de processos, uma vez que a otimização de metamodelos convergirá mais rapidamente.

\section{NOMENCLATURA}

cor Função de correlação

cov Covariância

E Esperança matemática

MV Variável Manipulável

P Parâmetros de processo

R Matriz de correlação

X Variável Independente

Y Resposta do modelo/metamodelo
$\mathbf{Z}$ Termo de correção

\section{Letras gregas}

$\boldsymbol{\beta}$ Coeficiente de regressão

б Variância

$\tau \quad$ Função de Regressão

\section{REFERÊNCIAS}

ALEX, J., BENEDETTI, L., COPP, J., GERNAEY, K. V., JEPPSSON, U., NOPENS, I., PONS, N., RIEGER, L., ROSEN, C., STEYER, J. P., VANROLLEGHEM, P., WINKLER, S. "Benchmark Simulation Model no. 1 (BSM1)," Department of Industrial Electrical Engineering and Automation, Lund University, Tech. Rep. CODEN:LUTEDX/(TEIE7229)/1-62/(2008), 2008.

FORRESTER, A., SOBESTER, A., KEANE, A. Engineering Design via Surrogate Modelling: A Practical Guide., John Wiley \& Sons, 2008.

GOMES, M.V.C., Otimização sequencial por aproximações - Uma aplicação em tempo real para o refino de petróleo. Tese de D.Sc., Universidade Federal do Rio de Janeiro, Rio de Janeiro, RJ, Brasil, 2007

JEPPSSON, U., NOPENS, I., BENNEDETTI, L., PONS, M.N., ALEX, J., COOP, J., GERNAEY, K.V., ROSEN, C., STEYER, J.P., VANROLLEGHEM, P. Benchmark simulation model No 2. Department of Industrial Electrical Engineering and Automation, Lund University, Tech. Rep. 2011

LOPHAVEN, S.N., NIELSEN, H.B., SONDERGAARD, J. 2002, DACE A MATLAB Kriging Toolbox, Technical Report IMM-TR-2002-12, version 2.0, Technical University of Denmark, Denmark, 2002

PALMER, K., REALFF, M. "Metamodeling Approach to Optimization of Steady-state Flowsheet Simulations - Model Generation", Trans IChemE, v. 80, Part A, pp. 760-772. 2002

SACKS, J., WELCH, J.W., MITCHELL, T.J. "Design and Analysis of Computer Experiments", Statistical Science., v.4, pp. 409-435. 1989

SANTNER, T.J., WILLIAMS, B.J., NOTZ, W.I. The Design and Analysis of Computer Experiments, New York, Springer-Verlag. 2003 